\title{
Thermally driven structural phase transformation and dislocation density of CdS nanoparticles precipitated without surfactant in $\mathrm{KOH}$ alkaline medium.
}

\author{
Chan Kok Sheng*, Yousef Mohammad Alrababah \\ Faculty of Science and Marine Environment, Universiti Malaysia Terengganu \\ 21030 Kuala Nerus, Terengganu, Malaysia. \\ *Corresponding author: chankoksheng@umt.edu.my
}

\begin{abstract}
The present research demonstrates a detailed discussion of the effect of annealing temperature on the structural transformation and surface morphology of the $\mathrm{CdS}$ nanoparticles synthesized using the precipitation method without surfactant in $\mathrm{KOH}$ alkaline medium. The annealing temperature used was in the range of $160-480^{\circ} \mathrm{C}$. The sample's structural, functional group, and morphological properties were investigated using XRD, FTIR, and SEM techniques. XRD analysis reveals that the $\mathrm{CdS}$ has gradually been transformed from the pure cubic to hexagonal polycrystalline structure and improved crystallinity upon increasing the temperature. Besides, the estimated crystallite size and dislocation density parameters were calculated using the established Debye-Scherrer equation. The average crystallite size was in nano-dimension and increased gradually with temperature. The FTIR spectra indicate that the characteristic vibration band of $\mathrm{CdS}$ emerged in the lower wavenumber region of 650 and $500 \mathrm{~cm}^{-1}$, and the band becomes stronger as the temperature rises. Also, the SEM images demonstrate that the CdS exhibits uniform spherical morphology, and the particle size grows more significant at elevated temperatures. The improved crystallinity and structural properties tuning ability against temperature allow beneficial optical applications as solar cells, photocatalysts, nonlinear optics, light-emitting diodes, and optoelectronic devices.
\end{abstract}

\section{Introduction}

Nowadays, nanotechnology has been applied in many fields, including semiconductor devices with better efficiency and tunability. The science and technology of nanomaterials have created great excitement and expectations in the last few years (Priya et al., 2018). In recent years, scientists discovered that the semiconductor materials in nanometer-size can exhibit different properties from those of the corresponding bulk semiconductors. This is because the quantum confinement effect present in the nano-sized semiconductor shows unique physical and chemical properties, such as size-dependent bandgap shifts (Alrababah et al., 2019; Dris et al. 2012; Sheng et al. 2017). CdS have become one of the magnetic materials among the II-IV group semiconductor compounds due to its direct wider optical bandwidth of $2.42 \mathrm{eV}$ at room temperature and the existing nonlinear optical properties (Abdel-Galil et al. 2017; Ayodhya et al. 2015; Hussein et al. 2019; Landeros et al. 2019; Martínez-Landeros et al. 2019; Shkir et al. 2020; Saxenaa et al. 2018; Yang et al. 2019). CdS nano-sized semiconductors have already been widely used as optical sensors, transistors, solar batteries, photo-electrocatalysis, as well as luminescence devices, laser, light-emitting diodes, 
detectors, and also in antibacterial activity (Göde, 2019; Hussein et al. 2019; Ji et al., 2019; Martínez-Landeros et al. 2019; Priya et al. 2018; Saxenaa et al. 2018; Sonker et al. 2020; Yang et al. 2019). CdS is also an n-type semiconducting material and acts as a window layer for solar cell fabrication due to its high value of transmittance and band gap width (Martínez-Landeros et al., 2019; Shkir et al. 2020).

Previously, tremendous attempts have been devoted to yielding nanoparticles with improved structural and physical properties (Yang et al., 2019). Hence, various physical and chemical methods have been proposed to produce the metal sulfides nanoparticles, such as the hydrothermal, solvothermal, precipitation, colloidal, sonochemical, and biosynthesis and sol-gel methods (Ayodhya \& Veerabhadram, 2019; Dris et al. 2013; Hussein et al. 2019; Mohamed et al. 2020; Priya et al. 2018; Sonker et al. 2020; Wilson \& Ahamed, 2019; Yang et al. 2019). The nanomaterial properties could be perfectly adjusted by the shape and morphology to meet the requirement and performance of the final product (Saxenaa et al., 2018). Therefore, it is reported that CdS nanostructure has been successfully synthesized with different morphologies of nanospheres, nanotubes, nanowires, hollow spheres, and flower-like structures (Yang et al., 2019). Among the mentioned methods, the precipitation method is considered a simple, effective, and economical route for the large-scale production of uniform-size and high-purity nanoparticles (Ji et al., 2019). Improved crystallinity and homogeneous grain size distribution would result in greater carrier mobility and, hence, superior device performance (Martínez-Landeros et al., 2019). This work synthesized the $\mathrm{CdS}$ nanoparticles via precipitation method in $\mathrm{KOH}$ alkaline solution and underwent further thermal annealing treatment at different temperatures. The impact of the annealing temperature on the structural and morphological modification was analyzed and discussed in detail. The crystallite size and dislocation density were determined from the Debye-Scherrer formula.

\section{Methodology}

\subsection{Preparation of CdS}

The chemical reagents employed in this work include cadmium chloride $(\mathrm{CdCl} 2)$, potassium hydroxide $(\mathrm{KOH})$, and thiourea, which were supplied by Merck company with analytical (AR) reagent grade (99.9\% purity), and they were used for sample preparation without any further purification. The aqueous solutions were prepared at room temperature and atmospheric pressure using deionized water. First, the $\mathrm{CdS}$ nanoparticles were synthesized through precipitation in $\mathrm{KOH}$ alkaline medium, in which the $\mathrm{CdCl}_{2}$ and thiourea precursors were used to generate the $\mathrm{Cd}^{+}$and $\mathrm{S}^{-}$ ion sources, respectively. First of all, $\mathrm{CdCl}_{2}(0.02 \mathrm{M})$, thiourea $(0.2 \mathrm{M})$, and $\mathrm{KOH}(0.5 \mathrm{M})$ were added into the deionized water to form an aqueous solution with a $\mathrm{pH}$ value of 13.82 . Next, the reaction mixture was then undergone continuous stirring in the water bath using a magnetic stirrer for 2 hours at $80{ }^{\circ} \mathrm{C}$ to obtain a homogeneous solution. After that, the initial colorless mixture solution was converted into a yellowish solution, indicating the formation of CdS precipitates. After that, the yellow deposits were separated from the solutions by filtration. Next, the sediments were alternately washed numerous times with distilled water to remove the unwanted impurities and then 
dried in atmospheric air at room temperature for one day. Finally, the products were annealed in an oven at different temperatures ranging from 160 to $480{ }^{\circ} \mathrm{C}$ with an interval increment of $160{ }^{\circ} \mathrm{C}$ for 3 hours to get the samples with different phases and purities. After annealing, the pieces were removed from the oven and left for natural cooling at $23{ }^{\circ} \mathrm{C}$ before further characterizations.

\subsection{Sample characterizations}

The crystalline phase and purity of the samples were characterized using the room-temperature Rigaku Miniflex-TM II X-ray diffractometer (XRD) with $\mathrm{Cu} K \alpha$ radiation $(\lambda=0.15406 \mathrm{~nm})$ generated at operating voltage of $40 \mathrm{KV}$ and $40 \mathrm{~mA}$ in the $2 \theta=20-80^{\circ}$ diffraction angle range at a scan speed of $0.02{ }^{\circ} \mathrm{s}^{-1}$. The Debye-Scherrer method was employed to calculate the mean crystallite size of the nanoparticles. The surface morphologies of the as-prepared samples were observed under the JSM JEOL-6360 scanning electron microscope (SEM) at an acceleration voltage of $15 \mathrm{kV}$. The functional groups of the pieces were identified by FTIR spectrophotometer (model: Thermo Nicolet Alvatar 380 FTIR). The IR spectrum was recorded at room temperature in the wavenumber region of $400-4000 \mathrm{~cm}^{-1}$ with $2 \mathrm{~cm}^{-1}$ spectral resolution.

\section{Results and discussion}

In this work, the crystalline structure of the CdS nanoparticles was characterized by utilizing the powder X-ray diffraction (XRD) technique. The XRD pattern of the CdS nanoparticles annealed at various temperatures is displayed in Figure 1. Naturally, CdS can exist in two different phases of cubic zinc-blende (hawleyite) and hexagonal wurtzite (greenockite) structures (Alrababah et al. 2019; Hussein et al. 2019; Priya et al. 2018). The multiple diffraction peaks presented in the XRD patterns signify the polycrystalline nature of the studied samples. From the Figure, it can be observed that the room-temperature synthesized $\mathrm{CdS}$ and the sample annealed at $160{ }^{\circ} \mathrm{C}$ shows three prominent diffraction peaks at $2 \theta \approx 26.34^{\circ}, 43.69^{\circ}$ and $51.89^{\circ}$, corresponding to lattice planes of (111), (220) and (311), respectively. These diffraction peaks are well-suited to a metastable CdS cubic structure (JPDS card file 75-1546) with lattice constant, $\mathrm{a}=5.82 \AA$, density $=4.866 \mathrm{~g} / \mathrm{cm}^{3}$, and space group (F-43m). Pure CdS is attained since no other peaks due to impurities are observed. Also, the cubic CdS possess the preferential orientation plane of (111) with a broad pattern of XRD line, which confirms the formation of $\mathrm{CdS}$ in nanoparticles size and can also indicate the amorphous behavior of the sample (Ji et al. 2019; Saxenaa et al. 2018). Moreover, the diffraction peaks become slightly broader for the sample annealed at $160{ }^{\circ} \mathrm{C}$, implying that finer $\mathrm{CdS}$ nanoparticles are produced at this temperature than those without annealing. This peak broadening could be due to the quantum size confinement and micro-straining of the crystal structure arising from the defects caused by a spontaneous growth of nanocrystals during a chemical reaction (Ayodhya \& Veerabhadram, 2019). Subsequently, the intensity of the diffraction peaks increases when the sample undergoes annealing treatment at $360^{\circ} \mathrm{C}$. At this temperature, it can be distinctly visualized in the XRD pattern that three stronger diffraction peaks emerged at $26.22^{\circ}, 43.52^{\circ}$ and $51.93^{\circ}$, which are respectively assigned to the (002), (110) and (112) planes, whilst four new peaks with relatively 
low intensity appeared at $24.85^{\circ}, 27.85^{\circ}, 36.65^{\circ}$ and $47.82^{\circ}$ corresponding to the scattering from (100), (101), (102) and (103) planes. All these diffraction peaks are well-indexed to the pure hexagonal wurtzite structure (JPDS card file 77-2306, $\mathrm{a}=\mathrm{b}=4.136 \AA$; $\mathrm{c}=6.713 \AA$; density $=4.881$ $\mathrm{g} / \mathrm{cm}^{3}$ and space group P63mc). Interestingly, this result also reveals that subsequent higher annealing temperature has induced the CdS to initially transform from the metastable cubic to hexagonal phase, which agrees with the previous work reported by Martínez-Landeros et al. (2019). Nevertheless, those weak peaks present in the XRD pattern could also plausibly conclude that the $\mathrm{CdS}$ is still in the intermediate phase consisting of a mixture of cubic and hexagonal structures (Saxenaa et al., 2018; Wilson and Ahamed, 2019). This means that the atomic displacement has started to rearrange its position at this temperature but has not yet been completed. As the annealing temperature increases to $480{ }^{\circ} \mathrm{C}$, all the seven peaks indexed to hexagonal structure become stronger and sharper than those prepared at lower temperatures. Again, this phenomenon indicates that the CdS nanoparticles are highly crystallized at elevated temperatures and are completely converted into a stable hexagonal phase (Ji et al. 2019; Yang et al. 2019). Hence, the collective atomic rearrangement to a more stable hexagonal structure is well established at this temperature. This structural conversion of the cubic CdS into pure hexagonal phase is also evidenced by Alrababah et al. (2019). A surfactant or complexing agent of ammonium nitrate is successfully utilized to tune the structure. Furthermore, the hexagonal CdS demonstrates the highest peak intensity at the (110) plane, implying the preferred orientation growth of the particles along this plane direction due to their lowest surface energy (Martínez-Landeros et al., 2019; Shkir et al. 2020). On the other hand, the $(002) /(111)$ plane seems more favorable for the cubic CdS obtained at lower annealing temperatures.

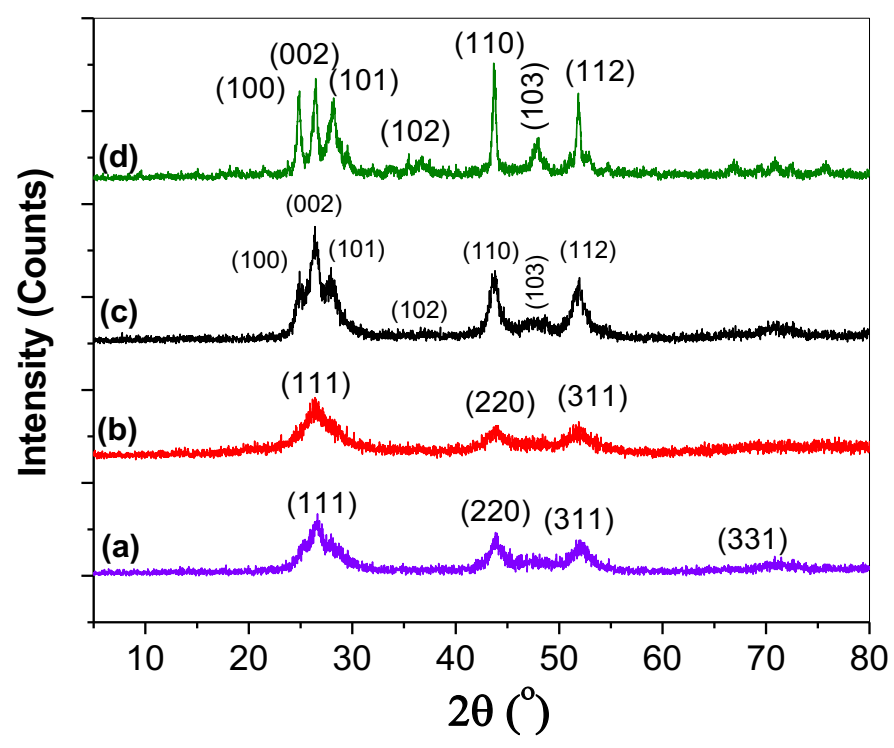

Fig. 1. XRD pattern of (a) as-synthesized $\mathrm{CdS}$ and the samples annealed at different temperatures: (b) $160{ }^{\circ} \mathrm{C}$, (c) $320^{\circ} \mathrm{C}$ and (d) $480{ }^{\circ} \mathrm{C}$. 
Henceforward, the Debye-Scherrer's equation was applied to compute the average crystallite size (D) of the CdS as given by $\mathrm{D}=0.9 \lambda / \beta \cos \theta$, where $\lambda$ represents the wavelength of $\mathrm{X}$-ray, $\beta$ is the FWHM of the diffraction peak, and $\theta$ is the peak angle of Bragg's diffraction (Alrababah et al. 2019; Göde et al. 2019; Kokaj et al. 2018; Othman et al. 2019; Sheng \& Alrababah, 2020). Afterward, the obtained $\mathrm{D}$ value is substituted in the following formula to acquire the mean dislocation density $(\delta)$ for the present samples (Shkir et al., 2020; Saxenaa et al., 2018).

$$
\delta=\frac{1}{\mathrm{D}^{2}}
$$

The calculated values of $\mathrm{D}$ and $\delta$ are displayed in Table 1 . The $\mathrm{D}$ values of the present samples are ascertained lying in the range of about $2-17 \mathrm{~nm}$. From the table, the average crystallite size of the as-synthesized sample is determined to be $3.21 \mathrm{~nm}$ for the cubic phase. This size shows a gradual reduction to $2.32 \mathrm{~nm}$ when the $\mathrm{CdS}$ is annealed up to $160{ }^{\circ} \mathrm{C}$, which could also be indirectly evidenced by the slight broadening of diffraction peaks with higher FWHM values. According to Shkir et al. (2020), a smaller crystallite size results in more significant grain boundaries formation that restricts the transference of the atoms or ions from one grain to another.

Nevertheless, the crystallite grows, and its average size increases as the $\mathrm{CdS}$ is annealed at a higher temperature up to $320^{\circ} \mathrm{C}$. This is because the atoms/ions in $\mathrm{CdS}$ have received excessive energy and started to rearrange in a more energetically stable and higher crystalline hexagonal structure. Upon further temperature increment up to $480^{\circ} \mathrm{C}$, the crystallite size continues to increase to a value of $16.75 \mathrm{~nm}$, accompanied by a highly crystalline hexagonal structure. At this stage, the structure formation with lattice expansion is induced by the highly energetic atoms, ions, or clusters with sufficient absorbed energy to construct the excellent structure. Equation (1) distinctively indicates that the dislocation density of the sample is inversely proportional to the square of crystallite size (Shkir et al. 2020). Thus, it is worth noting that the mean dislocation density reduces as larger crystallite size is produced, which might denote a decrease in the structural disorder of the sample when annealed at a higher temperature.

Table 1. Average crystallite size and dislocation density of CdS nanoparticles annealed at different temperatures.

\begin{tabular}{ccc}
\hline Samples & $\begin{array}{c}\text { Average crystallite size, D } \\
(\mathrm{nm})\end{array}$ & $\begin{array}{c}\text { Mean Dislocation Density, } \delta \\
\left(1 / \mathrm{nm}^{2}\right) \times 10^{-3}\end{array}$ \\
\hline As synthesized & 3.21 & 97.05 \\
$160^{\circ} \mathrm{C}$ & 2.32 & 185.79 \\
$320^{\circ} \mathrm{C}$ & 6.64 & 21.68 \\
$480^{\circ} \mathrm{C}$ & 16.75 & 3.56 \\
\hline
\end{tabular}


FTIR spectroscopy was used to verify the presence of the functional vibrational groups of the present materials. Figure 2 shows the FTIR spectra of the as-synthesized CdS and the CdS nanoparticles annealed at different temperatures, as measured in the $400-4000 \mathrm{~cm}^{-1}$ wavenumbers range. From the spectra, it could be observed that significant broadband occurred in the higher wavenumber region of 3600-2900 $\mathrm{cm}^{-1}$, which represents the hydroxyl group $(\mathrm{O}-\mathrm{H})$ stretching vibration of the water molecules absorbed on the sample surface as environmental moisture (Alrababah et al., 2019; Sheng et al. 2017). This band becomes weaker at elevated temperatures due to the evaporation of the water molecules from the surface of the particles. On the other hand, the small peaks located at $2346 \mathrm{~cm}^{-1}$ can be due to the stretching vibration of S-H bonds (Sonker et al. 2020). The absorption bands that appeared at around $1620 \mathrm{~cm}^{-1}$ might correspond to $\mathrm{CO}_{2}$ molecules attached to the particle surface during the characterization and synthesis process (Ayodhya et al., 2015). These two bands become smaller and finally diminish as the temperature rises, indicating the detachment of gas molecules from the particles.

Moreover, a moderate band positioned at $1100 \mathrm{~cm}^{-1}$ might be associated with the stretching mode of the sulfate group $\left(\mathrm{S}-\mathrm{O} / \mathrm{SO}_{4}\right)$. The characteristic band of $\mathrm{CdS}$ stretching vibration is observed at lower energy region between 650 and $500 \mathrm{~cm}^{-1}$ (Ayodhya \& Veerabhadram, 2019; Sonker et al. 2020; Alrababah et al. 2019; Sheng \& Alrababah, 2020). This band becomes slightly stronger at higher annealing temperature, which dictates that the atomic arrangement of $\mathrm{CdS}$ becomes much more ordered and would improve the IR transmission efficiency in the crystalline structure.

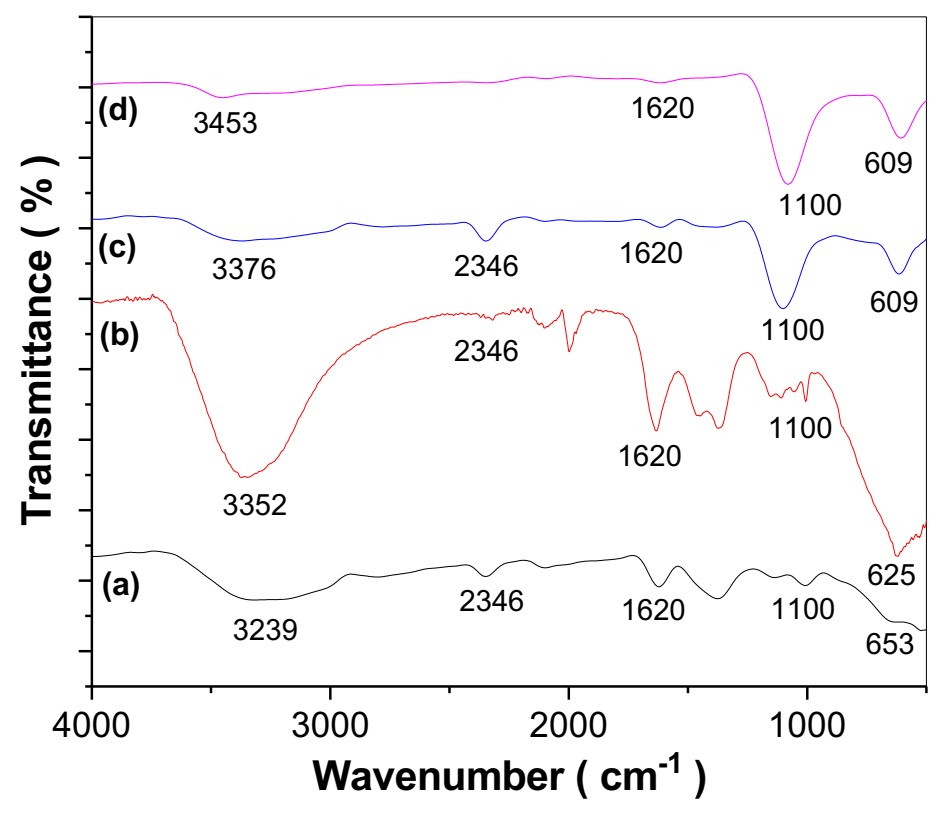

Fig. 2. FTIR spectra of (a) as-synthesized $\mathrm{CdS}$ and the samples annealed at different temperatures: (b) $160{ }^{\circ} \mathrm{C}$, (c) $320{ }^{\circ} \mathrm{C}$ and (d) $480{ }^{\circ} \mathrm{C}$. 
SEM characterized the surface morphology of the present materials. The SEM micrographs of the as-synthesized and thermally annealed CdS are illustrated in Figure 3. Clearly, the SEM images show that all the CdS nanoparticles possess uniform and dense spherical shapes with an average nano dimension of less than $100 \mathrm{~nm}$, contributing to a high surface area for specific applications. From the figure, the annealing temperature shows a significant effect on the surface morphology of the CdS nanoparticles. The as-synthesized sample shows an even and homogeneous spherical distribution with an average particle diameter of $\sim 70 \mathrm{~nm}$. The particle size is determined much larger than the crystallite size estimated from XRD data due to the reunion of many small nanocrystallites to construct a single particle. When the $\mathrm{CdS}$ is annealed with a heating temperature at $160{ }^{\circ} \mathrm{C}$, the particle size increases slightly, with the average diameter determined to be about $75 \mathrm{~nm}$.

Furthermore, as the sample is annealed up to $320^{\circ} \mathrm{C}$, the nanoparticles tend to agglomerate into the connected rock-salt-like structure with a particle size of around $80 \mathrm{~nm}$. However, it could be observed that a much better and more precise grain boundary is produced at this temperature, as also indicated in the Figure, which reflects an improvement in crystallinity for the hexagonal CdS as evidenced by XRD analysis. According to Hussein et al. (2019), the formation of aggregation is due to the coalescence of the nanoparticles as temperature increases. Henceforward, with a further rise in annealing temperature up to $480{ }^{\circ} \mathrm{C}$, we can see that the morphology of the $\mathrm{CdS}$ has transformed into a cluster-like structure, which comprises a larger particle size with an average diameter of $\sim 92 \mathrm{~nm}$. This cluster type possesses increased surface smoothness, leading to higher nucleation contact energy. In turn, it results in a well-established salt rock hexagonal structure formation with improved crystallinity (Saxenaa et al., 2018).
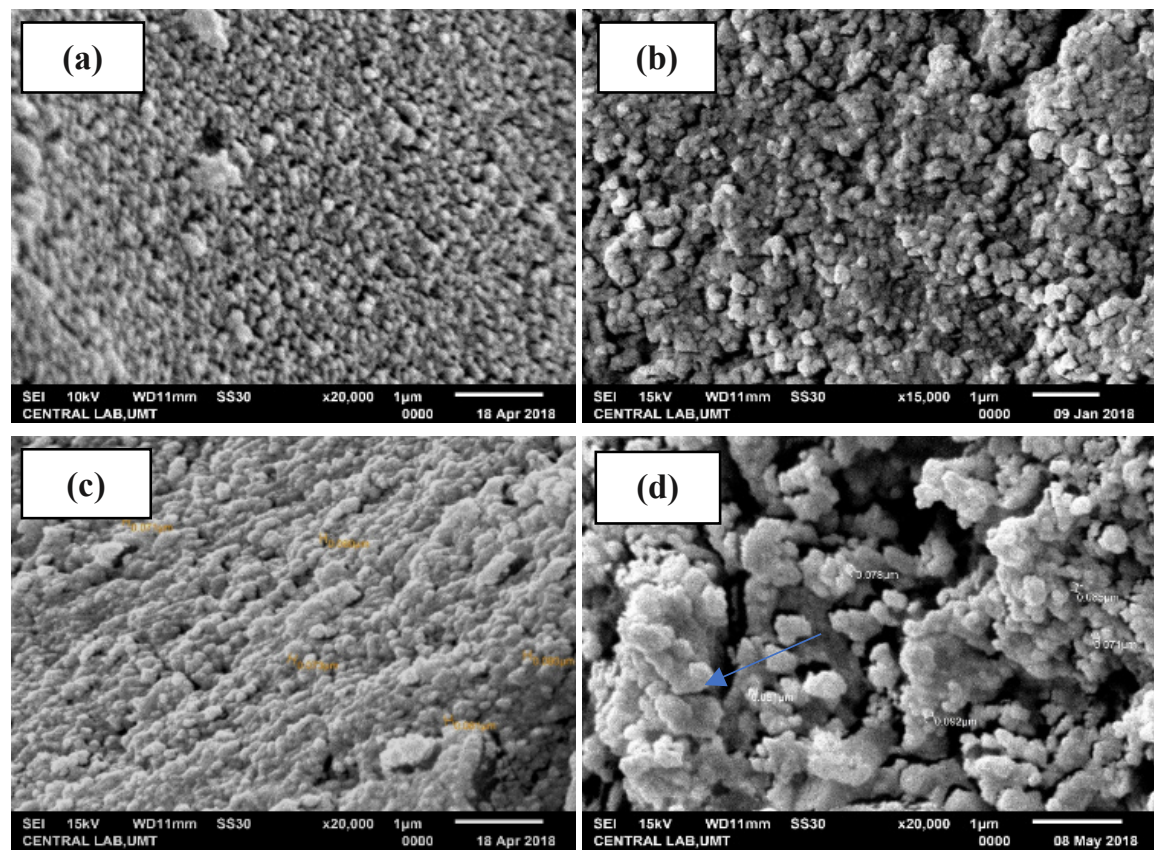

Fig. 3 SEM images of (a) as-synthesized $\mathrm{CdS}$ and the samples annealed at different temperatures: (b) $160{ }^{\circ} \mathrm{C}$, (c) $320^{\circ} \mathrm{C}$ and (d) $480{ }^{\circ} \mathrm{C}$. 


\section{Conclusions}

In summary, we have successfully investigated the effect of annealing temperature on the structural phase and morphological modifications of the CdS nanoparticles synthesized via a simple and lowcost precipitation method without any surfactant in the $\mathrm{KOH}$ solution. XRD, FTIR, and SEM characterized the samples. The as-synthesized $\mathrm{CdS}$ shows cubic phase formation. However, the crystalline structure is transformed from the cubic phase to the hexagonal phase with improved crystallinity when the sample is annealed at a higher temperature. Furthermore, the single-phase cubic and hexagonal structure shows the preferential peak orientation with high intensity and (111) and (002) directions, respectively.

Moreover, the average crystallite sizes were determined lying in the range of $3-15 \mathrm{~nm}$. The SEM analysis reveals that the samples are nearly spherical with nano-size dimensions. Besides, the average crystallite and particle sizes increase, accompanied by reduced mean dislocation density as the annealing temperature increases. From FTIR spectra, the peaks observed at around 650 and 500 cm-1 are related to metal sulphide vibrational bands, which confirms the formation of CdS nanoparticles in this study. Also, the water molecules organic or hydrogen bonds attached to the $\mathrm{CdS}$ particles become negligible with increasing annealing temperature, implying the formation of high-purity CdS nanoparticles. Eventually, thermally driven annealing temperature could play an important role in modifying and controlling the morphology and size of the CdS nanoparticles for desired applications.

\section{ACKNOWLEDGEMENTS}

The authors would like to thank the Faculty of Science and Marine Environment, Universiti Malaysia Terengganu, and the Malaysian Government for this work's technical and financial support through the research grant (TAPE-RG-55290).

\section{References}

Abdel-Galil A., Ali H.E., Balboul M.R. (2017). Influence of CdS nano-additives on the optical, thermal, and mechanical performance of CdS/polyvinyl alcohol nanocomposites. Optik, 129: 153162.

Alrababah Y.M., Sheng C.K., Hassan M.F. (2019). Influence of ammonium nitrate concentration on structural evolution and optical properties tuning of $\mathrm{CdS}$ nanoparticles synthesized by precipitation method. Nano-Structures \& Nano-Objects, 19: 10034.

Alrababah Y.M., Sheng C.K., Hassan M.F. (2019). Optical, structural, and morphological properties of CdS nanoparticles capped by ammonium nitrate in alkali medium by precipitation method. Chalcogenide Letters, 16(6): 297-301. 
Ayodhya D., Venkatesham M., Kumara A.S., Reddy G.B. Veerabhadram G. (2015). One-pot sonochemical synthesis of $\mathrm{CdS}$ nanoparticles: photocatalytic and electrical properties. International Journal of Industrial Chemistry, 6: 261-271.

Ayodhya D. \& Veerabhadram G. (2019). Facile fabrication, characterization, and efficient photocatalytic activity of surfactant-free $\mathrm{ZnS}, \mathrm{CdS}$, and $\mathrm{CuS}$ nanoparticles. Journal of Science: Advanced Materials and Devices, 4: 381-391.

Dris M.R.M., Sheng C.K., Isa M.I.N., Razali M.H. (2012). A study of cadmium sulphide nanoparticles with starch as a capping agent. International Journal of Technology, 1: 1-7.

Dris M.R.M., Isa M.I.N., Ismail M., Sheng C.K. (2013). Effects of Ethylene Glycol Concentration on Cadmium Sulfide Nanowires Growth. International Journal of Electroactive Materials, 1: 23-27.

Göde F. (2019). Effect of $\mathrm{Cu}$ doping on $\mathrm{CdS}$ as a multifunctional nanomaterial: Structural, morphological, optical, and electrical properties. Optik - International Journal for Light and Electron Optics, 197: 163217.

Hussein E.H., Mohammed N.J., Al-Fouadi A.H.A., Abbas K.N., Alikhan J.S., Maksimova K., Goikhman A.Y. (2019). Impact of deposition temperature on the structural properties of $\mathrm{CdS} / \mathrm{Si}$ nanoparticles for nanoelectronics. Materials Letters, 254: 282-285.

Ji.P.F. Li Y., Song Y.L., Zhou F.Q., Tian M.L., Yuan S.Q. (2019). Structure transition and photoluminescence of $\mathrm{CdS} / \mathrm{Si}$ nano heterostructure array on thermal annealing. Physics Letters A, 383: 125841 .

Kokaj J., Shuaib A., Makdisi Y., Mathew J. (2018). Femtosecond laser-based deposition of nanoparticles on a thin film and its characterization. Kuwait Journal of Science, 45: 37-45.

Martínez-Landeros V.H., Hernandez-Como N., Gutierrez-Heredia G., Quevedo-Lopez M.A., Aguirre-Tostado F.S. (2019). Structural, chemical, and electrical properties of CdS thin films fabricated by pulsed laser deposition using varying background gas pressure. Thin Solid Films, 682: 24-28.

Mohamed H.S.H., Rabia M., Shaban M., Taha S. (2020). Controlled synthesis of CdS nanoflowers thin films for $\mathrm{H}_{2}$ electro-generation. Materials Science in Semiconductor Processing, 120: 105307.

Othman K.I., Ali M. E. S., El-Hout S. (2019). Dielectric properties of sintered $\mathrm{BaTiO}_{3}$ prepared from barium acetate and titanium dioxide, Kuwait Journal of Science, 46: 53-59. 
Priya, N.S., Kamala, S.S.P., Anbarasu, V., Azhagan, S.A. Saravanakumar R. (2018). Characterization of $\mathrm{CdS}$ thin films and nanoparticles by a simple chemical bath technique. Materials Letters, 220: 161-164.

Saxenaa N., Kalsib T., Uttamb P., Kumarb P. (2018). Morphological evolution in nanocrystalline $\mathrm{CdS}$ thin films from flowers to rock-like salt structures. Optical Materials, 84: 625-630.

Sheng C.K., Amin K.A.M., Hong L.L., Hassan M.F., Ismail M. (2017). Investigation of morphological, structural, and electrical properties of Cds/ PMMA nanocomposite film prepared by solution casting method. International Journal of Electrochemical Science, 12: 10023-10031.

Sheng C.K. \& Alrababah Y.M. (2020). The Role of pH on Infrared Spectral, Structural and Morphological Properties of Room-temperature Precipitated CdS Nanoparticles. Journal of Nanoand Electronic Physics, 12(1): 01017.

Shkir M., Chandekar K.V., Khan A., Mohamed El-Toni A., Ashraf I.M., Benghanem M., Adil S.F., Ansari A.A., Ghaithan H., AIFaify S. (2020). Structural, morphological, vibrational, optical, and nonlinear characteristics of spray pyrolyzed CdS thin films: Effect of Gd doping content. Materials Chemistry and Physics, 255: 123615.

Sonker R.K., Yadav B.C., Gupta V., Tomar M. (2020). Synthesis of CdS nanoparticles by the sol-gel method as a low-temperature $\mathrm{NO}_{2}$ sensor. Materials Chemistry and Physics, 239: 121975.

Wang G.Z., Chen W., liang C.H., Wang Y.W., Meng G.W., Zang L.D. (2001). Preparation and characterization of $\mathrm{CdS}$ nanoparticles by ultrasonic irradiation. Inorganic Chemistry Communications, 4: 208-210.

Wilson K.C. \& Ahamed M.B. (2019). Influence of bath temperature on surface modification and optoelectronic properties of chemical bath deposited $\mathrm{CdS}$ thin film nanostructures. Materials Science \& Engineering B, 251: 114444.

Yang X., Yang Y., Wang B., Wang T., Wang Y., Meng D. (2019). Synthesis and photocatalytic property of cubic phase CdS. Solid-State Sciences, 92: 31-35.

Submitted: $\quad 22 / 01 / 2021$

Revised : $07 / 04 / 2021$

Accepted : $\quad$ 21/05/2021

DOI: $\quad 10.48129 / \mathrm{kjs} .11913$ 